VP-6

\title{
Minimally invasive living donor liver transplantation: Pure laparoscopic explant hepatectomy and graft implantation using upper midline incision
}

\author{
Kyung-Suk SUH ${ }^{*}$, Suk Kyun HONG, Kwangpyo HONG, Eui Soo HAN, Su young HONG, \\ Sanggyun SUH, Jeong-Moo LEE, YoungRok CHOI, Nam-Joon YI, Kwang-Woong LEE \\ Department of Surgery, Seoul National University Hospital, Korea
}

Introduction: Minimally invasive approaches have increasingly been applied in surgeries and have even recently been used in live donor hepatectomy. We have developed a safe and reproducible method for minimally invasive living donor liver transplantation (LDLT), which consists of pure laparoscopic explant hepatectomy and graft implantation using an upper midline incision.

Methods: From March 2020 to June 2020, minimally invasive LDLT was attempted in five patients. Explant hepatectomy was performed using the pure laparoscopic method, and graft implantation was performed using an upper midline incision.

Results: The procedure was completed in three of the five patients. The first two patients required open conversion due to bleeding. In the three successful procedures, the time required to remove the liver was 285, 180, and 166 minutes, respectively, and the total operative time was 640,575 , and 499 minutes, respectively. All patients recovered well, and the duration of hospital stay was 30 , 26, and 15 days, respectively.

Conclusions: According to the data of the present study, minimally invasive LDLT in properly selected recipients is a feasible and safe procedure when performed by a highly experienced surgeon and transplantation team. Further studies with larger series are needed to confirm the safety and feasibility. 